\title{
RELACIÓN PROFESIONAL SANITARIO - PACIENTE, DURANTE LA PANDEMIA DE COVID19. ¿DISTANCIAMIENTO SOCIAL O PROFESIONAL? ESTRATEGIAS ÉTICAS PARA EL FUTURO.
}

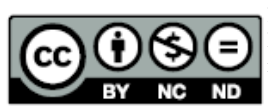

Este artículo está bajo una licencia de Creative Commons de tipo Reconocimien to - No comercial - Sin obras OPEN ACCESS derivadas 4.0 International

1 Universidad de Cuenca. Facultad de Ciencias Médicas. Posgrado de Medicina Legal y Forense.

Cuenca - Ecuador

ORCID ID:

Guarnizo Chávez Andrés

orcid.org/ 0000-0002-1578-2282

*Corresponding author: Guarnizo Chávez Andrés

E-mail: andresj.guarnizo@ucuenca.edu.ec

Received: $11-\mathrm{Jan}-2021$

Accepted: 22 - Feb - 2021

Publish: 01 - Mar - 2021

Conflict of interest: The author declared that there are no conflicts of interest.

Financial disclosure: The author have no financia relationships relevant to this article to disclose.

Forma de citar este artículo: Guarnizo A. RELACIÓN PROFESIONAL SANITARIO PACIENTE, DURANTE LA PANDEMIA DE COVID 19. ¿DISTANCIAMIENTO SOCIAL O PROFESIONAL? ESTRATEGIAS ÉTICAS PARA EL FUTURO. Rev Med Vozandes. 2021; 32 (1): .....- .......

\section{Resumen}

La pandemia de SARS Cov19 ha cambiado la forma de hacer medicina para siempre. De entre todas las disciplinas es quizá la bioética la que más debe adaptarse a los nuevos tiempos venideros. Las tecnologías indicadas para el entorno virtual de atención médica, las barreras de bioseguridad, y el distanciamiento social; han dejado huellas negativas en la relación médico paciente, la misma que se ha vuelto menos comunicativa y personal; para convertirse en algo más automatizado. Es hora de adecuar nuestras propias capacidades para mejorar y optimizar la atención sanitaria, El presente artículo trata sobre las limitaciones de la relación profesional sanitario - paciente en el contexto de la pandemia, y qué estrategias pueden implementar los profesionales y estudiantes para mejorarla.

Palabras clave: COVID-19, Relación médico paciente, Ética clínica, Bioética, Cuidado centrado en el paciente

\section{Abstract}

The SARS Cov19 pandemic has changed the way to make medicine forever. Of all the disciplines, it is perhaps bioethics that must best adapt to the new times ahead. The technologies indicated for the virtual healthcare environment, biosafety barriers and social distancing; has left negative marks on the doctor-patient relationship, which has become less communicative and personal; to become something more automated. It is time to adapt our own capacities to improve and optimize our care. This article deals with the limitations of the doctorpatient relationship in the context of the pandemic, and what strategies professionals and students can implement to improve it.

K eywords: COVID-19, Doctor patient relation, Clinical ethics, Bioethics, Patient centered care

\section{Introducción}

Una de las bases que garantizan la buena atención clínica, es la relación profesional sanitario - paciente, eje fundamental de la prestación sanitaria. La misma resume de manera aplicada y práctica, los principios de la llamada "bioética principalista" con la cual un profesional de salud tiene acercamiento desde las primeras cátedras de pregrado. Estos principios, de manera clásica se dividen por jerarquías:

-Principios de primer nivel: No maleficencia y justicia. Son obligatorios, exigibles por la sociedad mediante imposición de leyes y búsqueda de consenso.

-Principios de segundo nivel: Autonomía y Beneficencia. Obedecen al sistema de valores y se encuentran en la esfera privada e individual de las personas. (1)

La bioética ha sido quizá la parte más cambiante de la medicina y que ha evolucionado de mejor manera a lo largo de los tiempos; desde los modelos paternalistas clásicos donde el médico imponía su verdad 
y punto de vista, hasta el actual modelo de responsabilidad compartida donde tanto médico y paciente son entes activos de la toma de decisiones, cumpliendo con el tan mencionado principio de autonomía. (2)

En las facultades de medicina se instruye teóricamente la cátedra de bioética, y en la experiencia clínica y hospitalaria del estudiante, se la pone en práctica. Sin embargo, las nociones tradicionales de esta relación profesional sanitario - paciente han cambiado en el contexto de pandemia. Citamos el caso de la telemedicina donde estudiantes de los últimos semestres han sido designados para atender llamadas telefónicas a fin de orientar al usuario sobre los síntomas sospechosos del SARS Cov2, y a dónde acudir si éstos fueran positivos. Es decir, muchos estudiantes han tenido que enfrentarse a una nueva modalidad de relación profesional sanitario - paciente, sin haber tenido siquiera la experiencia necesaria en la modalidad clásica.

Los médicos ya formados, que han tenido la oportunidad de trabajar las vicisitudes propias de la atención personal; hoy se enfrentan a nuevos retos en la modalidad virtual o con exceso de barreras físicas entre ellos y sus respectivos pacientes.

¿Y qué hay del enfermo? Tampoco debemos olvidar que ellos, también acostumbrados a la atención médica tradicional, pueden verse consternados ante la imposibilidad de ver directamente a la persona que le trata su dolencia, al impedimento de acudir a un centro de salud por la cuarentena, - la simple idea que no está recibiendo la atención que se merece en base a sus necesidades.

Para los pacientes internos, la situación no es distinta: Tener que estar separados por áreas de aislamiento, evitar visitas de sus familiares, incluso el hecho que el profesional deba tocarlo lo "menos posible" y al hacerlo tener que llevar tantas barreras de bioseguridad; le dará la impresión de ser atendido por autómatas, y no por personas de carne y hueso tal como ellos. Pueden también auto percibirse como meros casos clínicos, como cifras o como enfermedades en lugar de seres humanos. El mismo caso se nota cuando por la gran afluencia de enfermos, se disminuye el tiempo de atención de cada uno. En un abrir y cerrar de ojos, pudieron haber llegado diez pacientes más, dejando un poco abandonado a los que ya se encontraban en espera de su consulta.

Una buena relación profesional sanitario- paciente, es aquella que beneficia a ambas partes. Al médico le recuerda el sentido mismo de su vocación, lo enaltece como persona y profesional; y al paciente le hace sentir seguro de encontrar la ayuda que requiere. Es saber conjugar la ciencia y el conocimiento, con valores humanos tales como amabilidad, servicio, y preocupación por el prójimo. (3)

\section{¿Cómo afecta la pandemia a la relación profesio- nal sanitario - paciente?}

En el libro "El médico y el enfermo" del autor Pedro Lain Entralgo, se citan de manera simultánea los cinco momentos fundamentales en la relación profesional sanitario - paciente, sin los cuales la misma no existiría. En base a esto se puede analizar con numerosos ejemplos como la emergencia sanitaria debilita cada uno de los eslabones necesarios en dicha relación.
1) Momento cognoscitivo: Cuando se llega al conocimiento nosológico de la enfermedad, sus causas, curso y consecuencias. Así como también del conocimiento de manera integral del paciente que la padece. ${ }^{(4)}$

Desde el inicio de la pandemia se priorizó en muchas instituciones al paciente COVID, relegando a segundo plano otro tipo de usuarios como por ejemplo aquellos de cirugías electivas, y postergando indefinidamente sus tratamientos. Pacientes oncológicos que se han complicado por no poder acudir a sus quimioterapias, y han tenido que buscar como alternativa la comunicación vía telefónica con sus especialistas. También se toman en cuenta los casos de subdiagnóstico en otras entidades patológicas, o complicación de las mismas.

2) Momento afectivo: Sentimientos $y$ emociones propios de la empatía entre médico y paciente. ${ }^{(4)}$

Ante una enfermedad de pronóstico incierto, y que ha generado un pánico social secundario, es inevitable encontrarnos con cuadros dramáticos sumamente emotivos, tales como médicos que deben comunicar a pacientes y familiares que su salud va empeorando, o incluso hasta el momento de dar las malas noticias.

3) Momento operativo: Como su nombre lo indica, corresponde al tratamiento y cualquier procedimiento que lleve a cabo el galeno, para tratar de curar a su paciente. Íntimamente ligado a la "lex artis". (4)

El enfermo y su familia tienen la percepción que su médico hará lo posible para realizar un tratamiento eficaz, lo que muchas veces se ve comprometido por la falta $y$ escases de recursos e insumos necesarios. A veces estas carencias que dependen de instancias administrativas superiores son vistas como error médico por personas poco comprensivas.

4) Momento ético: Implica el correcto cumplimiento de las normas que regulan la actividad de la medicina y la relación clínica. ${ }^{(4)}$

Al momento actual existe controversia en las guías clínicas y protocolos de Covid19, por la falta de consenso y evidencia de primer nivel. Prácticamente existen tantos lineamientos clínicos como hospitales en el mundo. 
5) Momento histórico social: Aspectos sociales del médico y del paciente en contexto del tiempo y lugar donde se desenvuelven. ${ }^{(4)}$

Es el más importante en la situación actual, pues nos encontramos frente a una situación que literalmente puso al mundo y sistema de salud "de cabeza" de un momento para otro, lo que conlleva a cambios bruscos en la atención.

\section{El perfil del médico y del estudiante de medicina}

La bioética en su sentido social más amplio impregna un nuevo modelo educativo que tiene en cuenta la dimensión social de los pacientes, así como su fisiología y psicología.5 Estas virtudes éticas de un buen profesional también se han visto comprometidas en el contexto de la atención cada vez menos personalizada.

Ya sea por distanciamiento frente a nuevas modalidades de atención, por la enfermedad en sí misma, por las barreras interpersonales que previenen contagios, o por la percepción subjetiva de un paciente que busca la mejor atención de manera desesperada; no se ha podido optimizar las relaciones humanas y la probidad que merecemos y a la que estamos acostumbrados.

Las universidades han tenido el deber histórico de formar médicos con un amplio compromiso humano y una serie de valores; los mismos que en contexto de pandemia no deben perderse, pero si adaptarse, incluso con una visión hacia el futuro postpandemia: ¿Cómo se modificará el pensum de estudios de los profesionales de la salud para ajustarse al nuevo ambiente epidemiológico? ¿Qué acciones de capacitación o de educación continua deben establecerse para esta nueva realidad? (6)

Es deber del profesional de la salud encontrar las mejores maneras de llegar a su paciente dentro de las posibilidades y restricciones que la situación lo permite. Esto sin quitar la responsabilidad compartida del paciente, que no debe limitarse como sujeto pasivo del acto médico, sino que también tiene el deber de cooperar, y muchas veces de comprender estas limitaciones, que van desde la cancelación de la mayoría de las consultas "no urgentes" con el fin de minimizar desplazamiento de pacientes hacia una institución, hasta complicaciones intradomiciliarias debido el miedo natural de los pacientes a acudir a un profesional cuando lo requieren. ${ }^{(7)}$

Siendo la humildad una característica fundamental del profesional, es momento de reconocer sus propias capacidades, y porque no sus propios límites. Aceptar que las más complejas especialidades ahora pasaron momentáneamente a segundo plano, y que, en aras de solventar las necesidades sanitarias durante esta pandemia, los más grandes especialistas se han convertido en médicos generales y hasta en tele-médicos.

El respeto al enfermo garantiza la buena relación profesional sanitario - paciente. Ésta va de la mano con la integridad moral que atestigua la igualdad de condiciones y derechos. La misma calamidad se ha encargado de ponernos en equivalencia, y a la vuelta de la esquina puede ser el personal de salud, los que postrados requieran alivio de sus síntomas.

Es el momento de ser veraces, para llegar a tener una buena confiabilidad con el usuario. Ser directos pero empáticos, ponerse en los zapatos del otro, y pensando que en cualquier momento uno de nosotros o nuestros seres queridos podrían estar en la misma situación. Analizar cómo nos gustaría ser tratados, y aplicarlo a nuestra práctica clínica; siempre de la mano con la mejor evidencia científica disponible y con la sinceridad del pronóstico esperado. Recordar que incluso la salud mental puede verse comprometida por una mala comunicación.

Autocontrol: El médico pasa como todos los seres humanos por sentimientos de angustia, impotencia, desesperación y ansiedad. Saber controlarse es parte fundamental de su desarrollo desde las aulas; para no menoscabar su propia salud mental y para que las personas a su alrededor no se vean afectadas por sus estados de ánimo. Esto nos lleva a otro punto: la valentía.

Un médico valiente podrá soportar muchas cosas; sin embargo, puede alcanzar el límite de estrés. Ver a diario contagios, situaciones críticas, fallecimiento de sus propios colegas; le hará cuestionarse tarde o temprano sobre su propia vulnerabilidad física o labilidad emocional, más aún si no se ha respetado el deber de conferir seguridad a los trabajadores que laboran en condiciones francamente riesgosas. ${ }^{\left({ }^{8}\right)}$ Muchos llegarán a preguntarse si solo es cuestión de tiempo que llegue su hora, si vale la pena arriesgarse por salvar a su prójimo. Y me atrevo a decir que la respuesta siempre será sí. Pues riesgo siempre hubo; cuando valoras una meningitis, cunado manejas material corto punzante, cuando vas en una ambulancia, etc. Que una nueva enfermedad no sea el pretexto para dejar la vocación de lado. Hay que ser mucho más estricto con las medidas de bioseguridad, sí; pero desistir, jamás.

\section{Retos futuros en la relación profesional sa- nitario - paciente}

La ética médica no es algo que viene implícito con el título, ni algo que se adquiere de noche a la mañana. Por el contrario; es algo que se debe cultivar en las mentes de los estudiantes en formación, a la vez que recordarles constantemente a los médicos ya graduados, para que no dejen de practicarla siempre en su vida profesional. Es deber de las facultades de ciencias de la salud en sus programas de pregrado y postgrado, así como a los hospitales, clínicas y centros de salud dentro de su auditoría interna; el capacitar sobre estos temas que muchas veces se pasan por alto.

Si la medicina es un arte en constante evolución, y la educación de la misma se adapta a los nuevos 
tiempos, la relación profesional sanitario - paciente no puede quedarse atrás.

\section{Telemedicina}

Si la atención médica virtual implementada para el distanciamiento obligatorio evita el contacto físico, se debe evitar ser solo una cara en la pantalla o una voz detrás del teléfono. Es fundamental demostrar que detrás de ese medio de comunicación existe una persona formada en normas humanísticas básicas. Se debe buscar nuevas estrategias comunicativas y a la vez empáticas, que hagan considerarse valorado al enfermo, y lo hagan sentir una persona real. Estrategias que pueden ir desde saludos personalizados, preguntas acerca de su entorno familiar o de la salud de sus seres queridos antes de entrar en la consulta misma. Una observación o una broma sutil que puedan generar ese ambiente de confianza necesario para que el paciente se sienta seguro y colabore con la atención.

\section{Consentimiento informado}

Una de las dificultades más notorias al momento de no tener una atención personal, es la obtención del consentimiento informado en casos que se requiera el mismo por escrito. Sin embargo, previo a dejar constancia en la historia clínica, es aceptable que para tales casos excepcionales se utilice consentimiento verbal por parte del paciente, y si es posible, grabar audio o video de dicha constancia. ${ }^{(9)}$

\section{Protocolos de atención}

Es normal encontrar en la práctica distintos tipos de pacientes, desde los colaboradores hasta los ansiosos, pasando por los expansivos y los despreocupados. Por tanto, si bien es imprescindible individualizar el trato para cada persona, es cierto también que al menos cada área clínica tenga un protocolo estandarizado para entrevistas tele médicas orientadas al usuario. Se recomienda usar un tono tranquilizador en las mismas, y adaptarse al ritmo de la conversación y las necesidades del paciente, utilizando un lenguaje accesible y empático con preguntas concretas que ayuden en el soporte tanto patológico como emocional. ${ }^{(10)}$

\section{Apoyo emocional}

Un gesto, una señal, unas palabras de aliento, o el simple hecho de poner la mano en el hombro de un paciente postrado en el ámbito hospitalario; puede ser la manera de hacerlo sentir mejor; de decirle "yo estoy aquí y haré lo posible por cuidarte". Llamar al paciente por su nombre hará que no se sienta como una estadística, un caso positivo, o un futuro deceso más.

\section{Comunicación}

Ya se ha hablado de la eventual escases de insumos, personal e infraestructura, entes necesarios para una atención oportuna y de calidad. La descompensación y subsiguiente muerte de un paciente crítico no es algo sorprendente, pero sí que en algunos casos podría haber sido evitable, ya sea con disponibilidad de respiradores, camas en $\mathrm{UCl}$, o médicos disponibles. Se ha evidenciado durante esta pandemia, que el antiguo dilema bioético de racionamiento de recursos vitales y a quien asignarlos, ha sido más fuerte que nunca; el decidir por ejemplo "a cuál de los dos pacientes asignar el único ventilador disponible" nunca ha sido fácil. Autores recomiendan maximizar los recursos, priorizar a los trabajadores de salud, no priorizar la asistencia de llegada y actuar en base a la evidencia científica disponible, etc. (11) Medidas que quizá al común de la gente o familiares les resulte difícil entender. La comunicación del estado, pronóstico, evolución, y desenlace es una prioridad

\section{Instrucción ética}

El papel del estudiante de medicina también es fundamental, aunque siempre con la reserva del caso. El apoyo asistencial a pacientes ambulatorios $u$ hospitalizados por otras causas puede ser su punto fuerte, así como servir de interlocutores entre pacientes ingresado que no pueden recibir visitas y sus familiares, etc. Aparte de los beneficios propios para el sistema de salud, también se podría obtener un beneficio personal para fortificar valores como altruismo y generosidad. (12)

Todo es cuestión de tener la voluntad, iniciativa, y ganas de entregar a la sociedad la bondad que caracteriza al galeno; y por la que decidió seguir esta profesión.

\section{Conclusiones}

La relación profesional sanitario - paciente es el pilar fundamental de la práctica de la medicina y como tal, debe seguir ejecutándose sin excepción en todos los ámbitos donde esta se lleve a cabo. Esta relación necesariamente debe ser bilateral y compartida donde tanto el galeno como el enfermo deberán escuchar y comprender al otro

Las universidades tienen la responsabilidad de formar profesionales con ética médica tanto en sus programas de pregrado como de postgrado, Mientras que las instituciones de salud deben hacerse cargo de fomentar su socialización y capacitación periódica.

En los tiempos de pandemia, el profesional sanitario debe adaptar sus conocimientos de bioética al contexto de la nueva forma de hacer medicina, tanto para no deshumanizar al arte de la misma, como para adaptarse e innovar estrategias que lleguen al paciente.

La telemedicina llegó para quedarse, tiene que verse e institucionalizarse como una nueva estrategia de atención, más que como una medida temporal. Por tanto dentro de la misma, es necesario adaptar un nuevo enfoque de la relación profesional de la salud - paciente, acorde con las necesidades informáticas, éticas y sobre todo, teniendo al usuario como centro de la atención sanitaria. 


\section{DILEMAS ÉTICOS}

\section{Referencias}

1.- Gracia D. Fundamentos de Bioética. $2^{a}$ ed. 7.- Pallarés Carratalá V, Górriz-Zambrano C, LlisMadrid: Triacastela; 2007.

2.- Arrubarrena $\vee$. La relación médico-paciente. Cir Gen. 201 1; 33(2):122-125.

3.- Arango P. The doctor-patient relationship. An ideal for the XXI century. Medicas UIS. 2012 25(1):63-69.

4.- Mendoza A. La relación médico paciente: consideraciones bioéticas. Rev Peru Gineco Obstet. 2017; 63(4):555-564

5.- Drane J. Bioethical Perspectives from Ibero-America. J Med Philosophy. 1996; 21 (6): 557-569.

6.- Guanche H, Suárez A, Márquez A, González A. Componente crítico en las estrategias de atención médica, prevención y control de la COVID-19. Educ Med Super. 2020; 34 (2): 45-51. terri Caro JL, Gorriz JL. La pandemia por la COVID-19: una oportunidad para cambiar la forma de atender a nuestros pacientes [The COVID-19 pandemic: An opportunity to change the way we care for our patients]. Semergen. 2020 Aug;46 Suppl 1:3-5.

8.- Alarcón D. Perspectivas éticas en el manejo de la pandemia COVID-19 y de su impacto en la salud mental. Rev Neuropsiquiatr. 2020; 83 (2): 97-103.

9.- Arimany-Manso J, Martin-Fumadó C. Medicolegal issues regarding from the COVID-19 pandemic. Med Clin (Engl Ed). 2020 Oct 23;155(8):344-346.

10.- Lopez J, et al. Soporte telefónico al paciente con EPOC en tiempos de la COVID-19. Open Respir Arch. 2020: 2 (3): 179 - 185.
11.- Fumadó C, Gómez E, Morlans M. Consideraciones éticas y médico-legales sobre la limitación de recursos y decisiones clínicas en la pandemia de la COVID-19. Rev Esp Med Legal; 2020; 46 (3):1 19-126.

12.- Milläin NÃ̃$\tilde{A} \pm e z-C o r t \tilde{A} @ s \quad J . ~ C O V I D-19$ por SARS-Cov2 tambi $\cong$ @n ha afectado a la Educaci $\tilde{A}^{3} n \quad M \tilde{A} @$ dica [COVID-19 due to SARS-Cov2 has also affected Medical Education]. Educacio'n Me'dica. 2020 July-August:21 (4):261-4. Spanish. 